\title{
Targeted gene disruption of matrix metalloproteinase-9 (gelatinase B) suppresses development of experimental abdominal aortic aneurysms
}

\author{
Robert Pyo, ${ }^{1}$ Jason K. Lee, ${ }^{1}$ J. Michael Shipley, ${ }^{2}$ John A. Curci,,${ }^{1}$ Dongli Mao, ${ }^{1}$ \\ Scott J. Ziporin, ${ }^{1}$ Terri L. Ennis, ${ }^{1}$ Steven D. Shapiro, ${ }^{2,3,4}$ Robert M. Senior, ${ }^{2,4}$ \\ and Robert W. Thompson $1,4,5$ \\ ${ }^{1}$ Department of Surgery, Section of Vascular Surgery, \\ ${ }^{2}$ Department of Internal Medicine, Division of Pulmonary and Critical Care Medicine, \\ ${ }^{3}$ Department of Pediatrics, Division of Allergy and Pulmonary Medicine, \\ ${ }^{4}$ Department of Cell Biology and Physiology, and \\ ${ }^{5}$ Department of Radiology, Washington University School of Medicine, Wohl Hospital, St. Louis, Missouri, USA \\ Address correspondence to: Robert W. Thompson, Section of Vascular Surgery, Washington \\ University School of Medicine, 9901 Wohl Hospital, 4960 Children's Place, St. Louis, Missouri 63110, USA. \\ Phone: (314) 362-7410; Fax: (314) 747-3548; E-mail: thompsonr@msnotes.wustl.edu.
}

Robert Pyo's present address is: Elmhurst Hospital Center, Department of Internal Medicine,

Mount Sinai Service, Elmhurst, New York, USA.

Received for publication November 17, 1999, and accepted in revised form April 7, 2000.

\begin{abstract}
Abdominal aortic aneurysms represent a life-threatening condition characterized by chronic inflammation, destructive remodeling of the extracellular matrix, and increased local expression of matrix metalloproteinases (MMPs). Both 92-kD gelatinase (MMP-9) and macrophage elastase (MMP-12) have been implicated in this disease, but it is not known if either is necessary in aneurysmal degeneration. We show here that transient elastase perfusion of the mouse aorta results in delayed aneurysm development that is temporally associated with transmural mononuclear inflammation, increased local production of several elastolytic MMPs, and progressive destruction of the elastic lamellae. Elastaseinduced aneurysmal degeneration was suppressed by treatment with a nonselective MMP inhibitor (doxycycline) and by targeted gene disruption of MMP-9, but not by isolated deficiency of MMP-12. Bone marrow transplantation from wild-type mice prevented the aneurysm-resistant phenotype in MMP-9-deficient animals, and wild-type mice acquired aneurysm resistance after transplantation from MMP-9-deficient donors. These results demonstrate that inflammatory cell expression of MMP9 plays a critical role in an experimental model of aortic aneurysm disease, suggesting that therapeutic strategies targeting MMP-9 may limit the growth of small abdominal aortic aneurysms.
\end{abstract}

J. Clin. Invest. 105:1641-1649 (2000).

\section{Introduction}

Abdominal aortic aneurysms (AAAs) are a common degenerative condition associated with aging and atherosclerosis (1). Although elective surgical repair is an effective approach to prevent deaths from ruptured AAAs, there is a conspicuous absence of alternative therapeutic strategies for this disease (2). Because human aneurysm tissues are characterized by destructive remodeling of the elastic media and outer aortic wall, recent investigations have emphasized disease mechanisms involving chronic aortic wall inflammation and the progressive degradation of fibrillar matrix proteins $(3,4)$.

Elastin is a major structural component of the aorta and one of the most durable proteins of the extracellular matrix (5). The dissolution of elastic fibers requires the presence of specific proteinases, and several elastolytic matrix metalloproteinases (MMPs) are thought to contribute to aneurysm development, including gelatinase A (72-kDa gelatinase; MMP-2), gelatinase B (92-kDa gelatinase; MMP-9), and macrophage elastase (MMP-12) (6-9). MMP-9 has attracted particular interest because it is the most abundant elastolytic proteinase secreted by human AAA tissue explants in vitro and because it is actively expressed by aneurysm-infiltrating macrophages located at the site of tissue damage in situ (8). MMP-9 expression also correlates with increasing aneurysm diameter (10), and it is elevated in the circulating plasma of patients with AAAs (11). MMP-12 is considered to play a similarly important role in aneurysm development, because it is selectively expressed by macrophages within AAA tissue, and, unlike other MMPs produced in the degenerating aortic wall, it is specifically localized to elastin fiber fragments by immunohistochemistry (9). These observations have led to speculation that either MMP-9 or MMP-12 might be necessary in aneurysmal degeneration, thereby providing targets for pharmacologic therapy $(2,4)$. 
Studies using animal models have led to important insights into the cellular and molecular events underlying aneurysmal degeneration (12). Many of these studies have involved an elastase-induced rat model of AAAs, in which a chronic aortic wall inflammatory response is associated with progressive elastic fiber degradation and aneurysmal dilatation $(13,14)$. Elastase-induced inflammation is accompanied by increased aortic wall production of MMPs (15), and treatment with antiinflammatory agents or MMP antagonists leads to preservation of medial elastin and a reduction in aneurysm development (16-19). These and other investigations have led to a conceptual model of aneurysm disease that emphasizes the role of inflammatory cellderived metalloproteinases, acting in a cascade, to mediate the degradation of aortic wall elastic fibers. An important but elusive goal has been to determine if one or more of these enzymes might be critical in aneurysmal dilatation. Because currently available MMP antagonists are not sufficiently specific to address this question, we developed a murine model of elastase-induced AAAs and used mice with targeted gene deficiencies to evaluate the relative importance of MMP-9 and MMP12 in aneurysm development.

\section{Methods}

Experimental animals. Wild-type 129/SvJ mice were obtained from The Jackson Laboratory (Bar Harbor, Maine, USA) and 129/SvEv mice were obtained from Taconic Farms (Germantown, New York, USA). MMP-9-/- and MMP-12-/- mice were originally developed by homologous recombination on $129 / \mathrm{SvEv}$ and $129 / \mathrm{SvJ}$ genetic backgrounds, respectively $(20,21)$. Double-deficient animals were generated for this study by interbreeding the MMP-9-/- and MMP-12//- strains. All experimental procedures were performed in male mice that had reached maturity (> 8 weeks of age).

Elastase perfusion and aortic diameter measurements. Anesthetized mice underwent laparotomy, and the abdominal aorta was isolated from the level of the left renal vein to the bifurcation (Figure 1). Preperfusion aortic diameter $(\mathrm{AD})$ was measured to a resolution of $0.01 \mathrm{~mm}$ with a calibrated ocular grid. Temporary silk ligatures were placed around the proximal and distal portions of the aorta, and an aortotomy was created at the bifurcation with a 30-gauge needle. Heat-tapered polyethylene tubing (PE-10; Baxter Healthcare Corp., McGraw Park, Illinois, USA) was introduced through the aortotomy and secured with a silk tie. Using a syringe pump calibrated to $100 \mathrm{mmHg}$ (Sage Instruments, Boston, Massachusetts, USA), the aorta was filled with saline containing $0.414 \mathrm{U} / \mathrm{mL}$ Type I porcine pancreatic elastase (specific activity $5 \mathrm{U} / \mathrm{mg}$ protein; E1250; Sigma Chemical Co., St. Louis, Missouri, USA). For controls, the elastase solution was heat-inactivated $\left(100^{\circ} \mathrm{C}\right)$ before use. The aorta typically dilated about 50 to $70 \%$ during the 5 -minute period of elastase perfusion, regardless of the solution instilled. The perfusion catheter was then removed

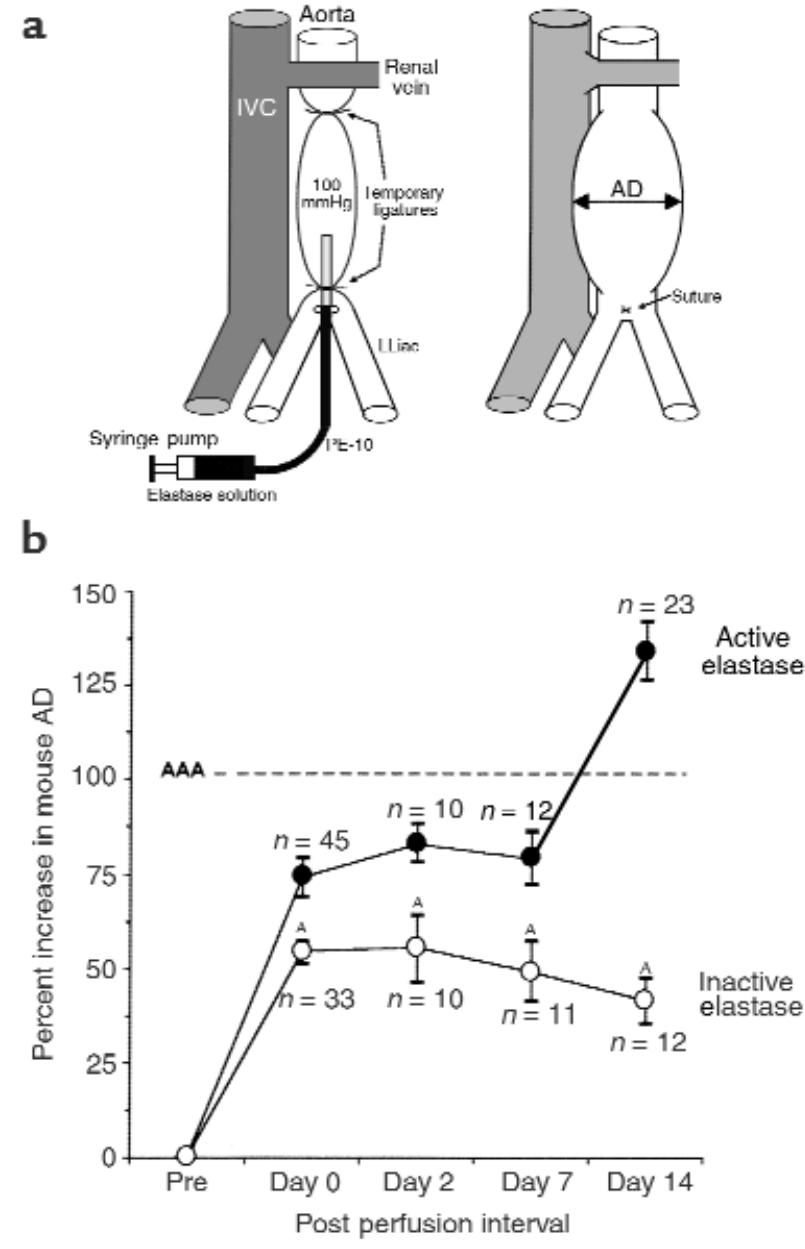

Figure 1

Development of elastase-induced abdominal aortic aneurysms in the mouse. (a) Schematic diagram of the elastase perfusion procedure, as described in Methods. IVC, inferior vena cava. (b) AD measurements following transient perfusion with elastase (filled circles) or heat-inactivated elastase (open circles), expressed as a percent increase between the preperfusion and final $A D(\triangle A D)$. Each point represents the mean \pm SEM (AP $<0.05$, Student's $t$ test). The incidence of AAAs ( $\triangle \mathrm{AD}>100 \%$; horizontal line) was significantly different between groups at 14 days $\left(P<0.05, \chi^{2}\right.$ analysis $)$.

and the aortotomy closed with a 10-0 suture to avoid constriction. Postperfusion AD was measured at least 5 minutes after restoration of lower-extremity perfusion, and the animals were allowed to recover for various intervals up to 14 days. The aorta was re-exposed by laparotomy, and final $\mathrm{AD}$ measurements were obtained before sacrifice. The percentage of increase in $\mathrm{AD}(\triangle \mathrm{AD})$ was calculated from the difference between the preperfusion and final $A D$, with $A A A s$ arbitrarily defined as $\triangle \mathrm{AD}$ more than $100 \%$. The mean plus or minus the SEM of group data were compared by ANOVA and the Student-Newman-Keuls multiple comparisons test or by the Student's $t$ test for paired analyses, and the prevalence of AAAs in each group was compared by $\chi^{2}$ analysis. 
Table 1

Effect of doxycycline on the development of elastase-induced AAAs

\begin{tabular}{|c|c|c|c|c|c|c|}
\hline \multirow[b]{2}{*}{ Treatment } & \multirow[b]{2}{*}{$n$} & \multicolumn{3}{|c|}{ Mouse aortic diameter $(\mathrm{mm})^{A}$} & \multirow{2}{*}{$\begin{array}{c}\Delta \mathrm{AD}(\%)^{\mathrm{B}} \\
14 \text { days }\end{array}$} & \multirow{2}{*}{$\begin{array}{c}\text { AAA }(\%) \\
14 \text { days }\end{array}$} \\
\hline & & pre-elastase & postelastase & 14 days & & \\
\hline None & 15 & $0.95 \pm 0.02$ & $1.70 \pm 0.05$ & $2.21 \pm 0.08$ & $134 \pm 9$ & 93 \\
\hline Doxycycline $e^{\mathrm{D}}$ & 8 & $1.00 \pm 0.03$ & $1.73 \pm 0.07$ & $1.91 \pm 0.21^{\mathrm{E}}$ & $89 \pm 17 \mathrm{E}$ & $50^{\mathrm{F}}$ \\
\hline
\end{tabular}

${ }^{A} A D$ measurements were obtained immediately before (pre) and after (post) elastase perfusion and 14 days later. ${ }^{B}$ The increase in aortic diameter ( $\triangle A D$ ) was expressed as a percent compared with the pre-elastase baseline. CPercentage of individual animals developing AAAs $(\triangle A D>100 \%)$. ${ }^{D}$ Animals treated with doxycycline beginning the day after elastase perfusion $(30 \mathrm{mg} / \mathrm{kg}$ per day supplied in the drinking water). All data represent the mean \pm SEM (EP<0.05, Student's $t$ test; ${ }^{F} P<0.05, \chi^{2}$ analysis).

Light microscopy and immunohistochemistry. Abdominal aortic tissues were perfusion-fixed with $10 \%$ neutral buffered formalin and embedded in paraffin, then cut in cross-sections $(5-10 \mu \mathrm{m})$ and stained with Verhoeff-van Geisen (VVG) for elastin. For immunohistochemistry, endogenous peroxidase activity was quenched with $3 \% \mathrm{H}_{2} \mathrm{O}_{2}$ for 30 minutes, and sections were blocked with dilute rabbit or goat serum. Slides were incubated overnight $\left(4^{\circ} \mathrm{C}\right)$ with one of the following primary antibodies (1:500): monoclonal rat anti-mouse Mac3 for macrophages (PharMingen, San Diego, California, USA); a monoclonal rat anti-mouse IgG recognizing a $40-\mathrm{kDa}$ neutrophil-specific cell surface antigen (MCA771G; Harlan/Serotec Inc., Indianapolis, Indiana, USA); and an antiserum to murine MMP-9 made in rabbits by immunization with recombinant active domain of murine MMP-9 (T. Betsuyaku, Y. Fukada, W.C. Parks, J.M. Shipley, and R.M. Senior, manuscript submitted). Control sections were incubated with affinitypurified rat $\operatorname{IgG}_{2 b} \kappa$ isotype (PharMingen) or normal rabbit IgG (Vector Laboratories Inc., Burlingame, California USA). Biotin-conjugated secondary antibodies were applied for 30 minutes at $25^{\circ} \mathrm{C}$ (mouse- adsorbed rabbit anti-rat IgG for macrophages and neutrophils or goat anti-rabbit IgG for MMP-9), and immune complexes were detected by avidin-biotinperoxidase with $3,3^{\prime}$-diaminobenzidine (Vectastain Elite kit; Vector Laboratories Inc.). Slides were counterstained with hematoxylin.

Substrate zymography. Protein extracts of aortic tissue were prepared as described (9), and samples $(10 \mu \mathrm{g})$ were resolved by nonreducing $10 \%$ SDS-PAGE through gels containing $1 \mathrm{mg} / \mathrm{mL}$ gelatin or casein (8). Gels were washed with Triton X-100 to remove the SDS, then incubated overnight $\left(37^{\circ} \mathrm{C}\right)$ in $50 \mathrm{mM}$ Tris- $\mathrm{HCl}$, $\mathrm{pH}$ 8.5, $5 \mathrm{mM} \mathrm{CaCl}_{2}$, and $0.5 \mathrm{mM} \mathrm{ZnCl}_{2}$. Zones of lysis were visualized after staining the gels with $0.5 \%$ Coomassie blue R-250.

RT-PCR/Southern blot analysis. Aortic tissue samples were pulverized under liquid nitrogen, and total RNA was isolated with Trizol reagent (GIBCO BRL, Grand Island, New York, USA) (9). First-strand cDNA synthesis was performed using $0.5 \mu \mathrm{g}$ of total RNA and $50 \mathrm{U}$ murine Moloney leukemia virus reverse transcriptase (GeneAmp RNA PCR kit; Roche Molecular Systems, Inc., Branchburg, New Jersey, USA), with all reactions performed in duplicate on a Gene AMP 2400 PCR ther-
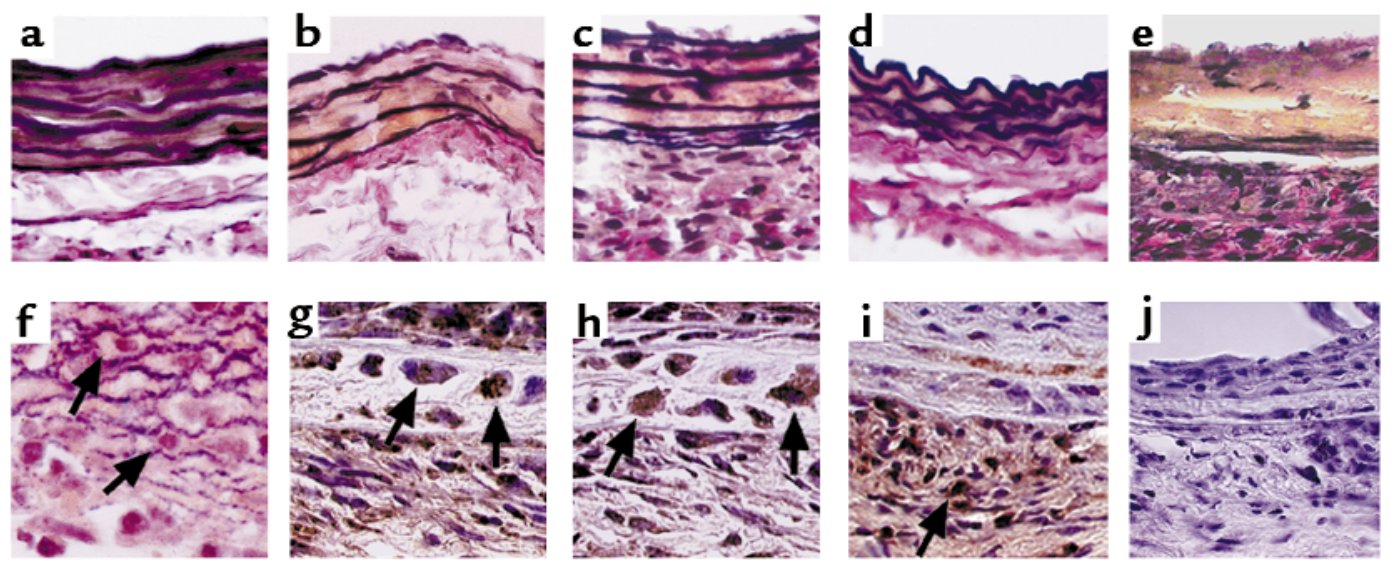

\section{Figure 2}

Histologic changes after elastase perfusion. Cross-sections of the aortic wall are shown with the lumen at the top. (a-f) Sections stained with WG for elastin. Normal preperfusion aorta (a), heat-inactivated elastase 7 days after perfusion (b), active elastase 7 days after perfusion (c), heat-inactivated elastase 14 days after perfusion (d), active elastase 14 days after perfusion (e), and active elastase 14 days after perfusion with fragmented elastic fibers surrounding mononuclear phagocytes (arrows) (f). (g-j) Active elastase 14 days after perfusion, with immunoperoxidase staining for macrophages $(\mathbf{g})$, MMP-9 (h), neutrophils (i), and IgG (negative control) (j). Positive-staining cells are indicated by arrows. $\times 100$. 


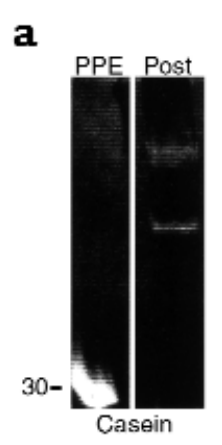

b

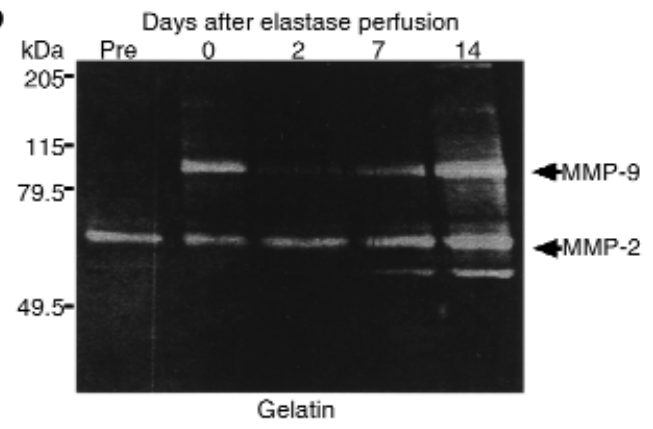

c

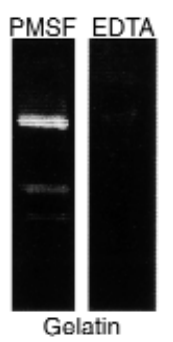

d

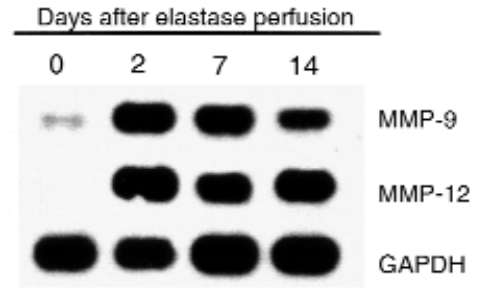

mal cycler system from Perkin-Elmer Corp. (Norwalk, Connecticut, USA). The reaction products served as the template for 30 cycles of PCR amplification (1 minute at $95^{\circ} \mathrm{C}, 1$ minute at $55^{\circ} \mathrm{C}$, and 1 minute at $72^{\circ} \mathrm{C}$, and 7 minutes at $72^{\circ} \mathrm{C}$ for final extension), using the following forward primer (FP) and reverse complement primer (RCP) pairs: mouse MMP-9 (GenBank reference Z27231), FP 5'-TAGTGAGAGACTCTACACAG-3' (bp 835-854), RCP 5'-CCACTTCTTGTCAGTGTCGA-3' (bp 1155-1174); mouse MMP-12 (GenBank reference M82831), FP 5'-ACAGCATCTTAGAGCAGTGC-3' (bp 270-289), RCP 5'-AGTCCACGTTTCTGCCTCAT-3' (bp 572-591); and mouse GAPDH (GenBank reference M32599), FP 5'-ATGTCGTGGAGTCTACTGGT-3' (bp 321-340), RCP 5'-TGGCATGGA CTGTGGTCATG-3' (bp 562-581). For mice with targeted disruption of MMP9 , alternate primers were used to amplify a specific sequence from within the disrupted region: FP 5'TGGCA GAGGCATACTTGTAC-3' (bp 153-172) and RCP 5'-AACTGGATGACAATGTCCGC-3' (bp 509-528). RTPCR products were resolved by agarose electrophoresis and transferred to Hybond $\mathrm{N}^{+}$nylon membranes (Amersham Life Science Inc., Arlington Heights, Illinois, USA). Using an enhanced chemiluminescence (ECL) 3 '-oligolabeling and detection system purchased from Amersham, Southern hybridization was performed with oligonucleotide probes specific to mouse MMP-9 (5'-ACCACAGCCAACTA TGACCA-3' [bp 965-984] or 5'-CCTCAAGTGGGACCATCATA-3' [bp 358-377] for knockout mice), MMP-12 (5'-GGTACCTCACTTACAGGATC-3' [bp 314-333]), and GAPDH (5'-ATGG GTGTGAACCACGAGAA-3' [bp 437-456]). Membranes were washed under stringent conditions, then incubated with horseradish peroxidase-conjugated anti-fluorescein antibodies and ECL kit reagents.

\section{Figure 3}

MMP production during aneurysm development. (a) Casein zymography of porcine pancreatic elastase (PPE) and mouse aortic wall extract obtained 24 hours after elastase perfusion (post). (b) Gelatin zymography depicting the pro- and activated forms of MMP-2 and MMP-9 (kDa, mobility of molecular weight standards). (c) Gelatin substrate zymogram (14 days after elastase sample) incubated in substrate buffers containing $5 \mathrm{mM}$ PMSF or 10 mM EDTA. (d) RT-PCR/Southern blots to detect MMP-9 and MMP-12 mRNA, with GAPDH as a loading control.

Irradiation and bone marrow transplantation. 129/SvEv wild-type and MMP-9-/- mice underwent lethal gamma irradiation ( $9.5 \mathrm{~Gy})$ from a cesium source, followed 6 hours later by transplantation with femoral bone marrow cells obtained from 6-to10-week-old syngeneic $\mathrm{SvEv}$ donors $\left(5 \times 10^{6}\right.$ cells per recipient; $0.3-0.5 \mathrm{~mL}$ by intravenous injection). Four groups were studied: (a) wild-type marrow transplanted to wild-type recipients; (b) wildtype marrow transplanted to MMP-9-/- recipients; (c) MMP-9-/- marrow transplanted to wild-type recipients; and (d) MMP-9-/- marrow transplanted to MMP-9-/- recipients. Mice were housed in a specific-pathogen-free-barrier environment, and all nontransplanted animals died within 14 days of irradiation. Animals achieving successful engraftment underwent the elastase perfusion procedure at least 10 weeks after transplantation.

\section{Results}

Development of an elastase-induced mouse model of aortic aneurysm. Our initial goal was to develop a reproducible murine model of elastase-induced AAAs for application to gene-targeted animals. Measurements of AD were obtained in 45 adult male $129 / \mathrm{SvJ}$ mice before and after transient intraluminal perfusion with porcine pancreatic elastase, with 33 animals undergoing perfusion with heat-inactivated elastase as controls (Figure 1). A moderate amount of aortic dilatation was present immediately after perfusion, with AD increasing after elastase $(74 \pm 5 \%)$ and after inactive elastase $(54 \pm 3 \%)$. Although this initial difference in diameter was significant $(P<0.05)$, there was no further increase in $\mathrm{AD}$ in either group up to 7 days, and the increase in $\mathrm{AD}$ did not reach aneurysmal proportions $(\triangle \mathrm{AD}>100 \%)$ either immediately after perfusion or within 7 days of perfusion. In contrast, the difference in $\mathrm{AD}$ between groups diverged markedly by 14 days, with the development of AAAs in 21 of 23 animals (91\%) perfused with elastase, but no aneurysms in 12 control animals perfused with inactive elastase (mean $\triangle \mathrm{AD}, 134 \pm 8 \%$ vs. $41 \pm 6 \%$; $P<$ 0.05 ). These findings establish that transient elastase perfusion is initially accompanied by only moderate aortic dilatation up to 7 days, but that AAAs consistently develop by 14 days. 
Light microscopy revealed minimal damage to the medial elastic lamellae for up to 7 days in both experimental groups (Figure 2, a-c). At the 14-day interval associated with aneurysmal dilatation, elastase-perfused aortas exhibited a dense inflammatory response and extensive degradation of medial elastin; in contrast, the elastic lamellae appeared nearly normal in animals perfused with heat-inactivated elastase (Figure 2, d and e). Fragmented elastic fibers were observed frequently surrounding mononuclear phagocytes in elastase-induced aneurysm tissues (Figure 2f), and leukocytes reactive with a macrophage-specific antigen (Mac3) were the dominant cell type detected by immunohistochemistry. These cells were frequently colocalized with immunoreactive MMP-9 on serial sections, but MMP9 did not appear in association with medial smooth muscle cells or fibroblasts (Figure 2, g and h). Although polymorphonuclear neutrophils (PMNs) were also present in elastase-induced AAA tissues, they were distributed in the peripheral aspect of the aortic adventitia rather than the degenerating media, and their pattern was distinct from that of immunoreactive MMP-9 (Figure 2i). These observations demonstrate that the development of elastaseinduced aneurysms is temporally and spatially associated with a chronic transmural inflammatory response, destructive changes in aortic wall elastin, and inflammatory cell production of MMP-9.

Porcine pancreatic elastase $(\sim 30 \mathrm{kDa})$ was not detectable in aortic wall extracts within 24 hours of elastase perfusion (Figure 3a), indicating that proteinases other than pancreatic elastase are responsible for the late degradation of aortic wall elastin associated with aneurysmal dilatation. The only gelatinase produced in the normal aorta was MMP2 (Figure $3 b$ ), and within 2 days of elastase perfusion there was a slight increase in this enzyme along with the appearance of its lower-molecular-weight (activated) form. MMP-9 ( 105 kDa) was also induced at this early interval, and activated MMP-2 and MMP-9 were both increased further by 7 days and particularly by 14 days, when the elastase-perfused aortas underwent aneurysmal dilatation. Using RT-PCR coupled with Southern blots, aortic wall expression of MMP-9 and MMP-12 mRNA was also increased during the development of elastaseinduced AAA (Figure 3d).

The functional significance of MMPs in elastaseinduced AAAs was examined in mice treated with doxycycline, a nonselective MMP inhibitor, for 14 days after elastase perfusion (Table 1). Doxycycline-treated animals exhibited a significant reduction in mean $\mathrm{AD}$ compared with untreated controls, as well as a reduction in the incidence of AAAs in individual animals, from 91 to $50 \%(P<0.05)$. These observations collectively support the notion that MMPs are responsible for the progressive connective tissue degradation that characterizes this model of AAAs.
Suppression of aneurysm development in gene-targeted mice. To examine the possibility that either MMP-9 or MMP-12 might be required in the development of experimental AAAs, we examined the response to elastase perfusion in mice with targeted disruption of the MMP-9 and MMP-12 genes. While phenotypic and functional abnormalities in MMP-9-/- and MMP-12-/mice have been described (20-22), neither of these animals is known to exhibit spontaneous cardiovascular

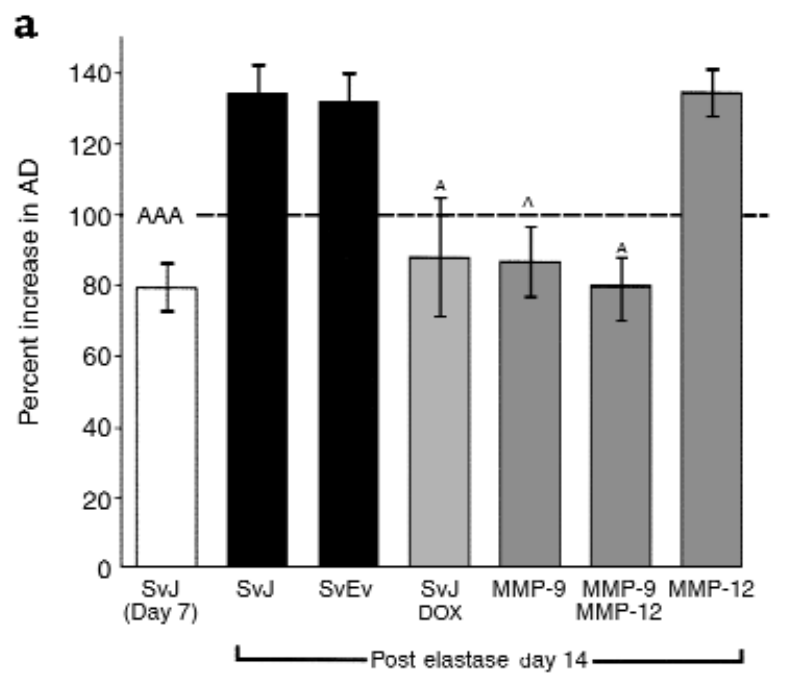

b

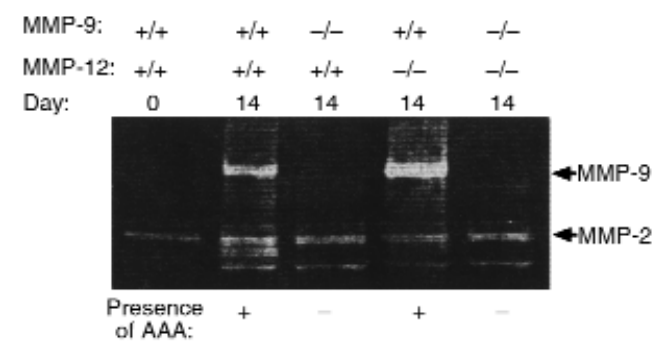

C

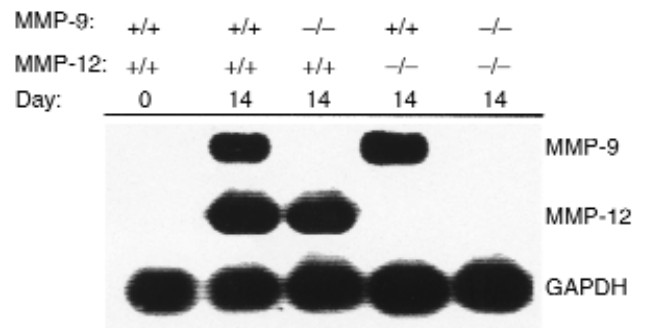

Figure 4

Effect of targeted gene deletions on elastase-induced aneurysmal dilatation. (a) The percent increase in $\mathrm{AD}$ was determined after 7 days (129/SvJ negative control; $n=12$ ) or 14 days (all other groups). The $129 / \mathrm{SvJ}(n=23)$ and 129/SvEv $(n=10)$ mice served as wild-type background controls for MMP-12-/- $(n=10)$ and MMP-9-/- $(n=10)$ animals, respectively, as well as double-deficient (MMP-9-/-/MMP-12-/-) mice $(n=10)$. Dox indicates $129 /$ Sv wild-type mice treated with doxycycline for 14 days after elastase perfusion $(n=8)$. Data shown represent the mean \pm SEM for each group ( ${ }^{A} P<0.05$ compared with the 14-day SvJ control group). (b) Gelatin zymography depicting MMP-2 and MMP-9 production in wild-type and gene-targeted mice. (c) RT$\mathrm{PCR} /$ Southern blots demonstrating the expression of MMP-9 and MMP-12 in the same animals, with GAPDH as a loading control. 
Table 2

Effects of bone marrow transplantation on elastase-induced aneurysmal dilatation

Mouse aortic diameter $(\mathrm{mm})^{\mathrm{A}}$

\begin{tabular}{llcccc} 
Donor & $\rightarrow$ Host $^{\mathrm{B}}$ & $n$ & pre-elastase & postelastase & 14 days \\
Wild-type & $\rightarrow$ Wild-type & 7 & $0.50 \pm 0.01$ & $0.83 \pm 0.02$ & $0.90 \pm 0.04$ \\
MMP-9-/- & $\rightarrow$ Wild-type & 10 & $0.52 \pm 0.01$ & $0.87 \pm 0.02$ & $0.79 \pm 0.02^{\mathrm{C}}$ \\
MMP-9-/- & $\rightarrow$ MMP-9-/- & 9 & $0.52 \pm 0.01$ & $0.83 \pm 0.02$ & $0.78 \pm 0.02$ \\
Wild-type & $\rightarrow$ MMP-9-/- & 12 & $0.50 \pm 0.01$ & $0.87 \pm 0.02$ & $1.00 \pm 0.03 \mathrm{C}$ \\
\hline
\end{tabular}

${ }^{A} A D$ measurements were obtained immediately before (pre) and after (post) elastase perfusion and 14 days later. ${ }^{B} 129 / \mathrm{SvEv}$ mice underwent lethal irradiation and syngenic bone marrow transplantation as indicated, 10 weeks before undergoing elastase perfusion. All data represent the mean \pm SEM ( ${ }^{C} P<0.05$, Student's $t$ test).

abnormalities. To guard against the possibility that MMP-9 and MMP-12 might be biologically redundant with respect to their elastolytic activities, we also examined mice with targeted disruption of both MMP-9 and MMP-12. Before the elastase perfusion experiments, we detected no discernible difference in aortic wall structure between any of these mutant strains and their wild-type counterparts (data not shown).

Elastase perfusion experiments revealed that aneurysmal dilatation is substantially reduced in MMP-9-deficient mice and in animals with combined deficiency in both MMP-9 and MMP-12 (Figure 4a). Thus, the increase in aortic diameter by 14 days after perfusion was only $87 \%( \pm 10 \%)$ in MMP-9-/- mice and $79 \%( \pm 9 \%)$ in MMP-9-/-/MMP-12-/- mice, compared with $134 \%$ $( \pm 8 \%)$ and $132 \%( \pm 8 \%)$ in the $129 / \mathrm{SvJ}$ and $129 / \mathrm{SvEv}$ background controls, respectively $(P<0.05$, Student's $t$ test). AAAs developed in $40 \%$ of the MMP-9-/- animals and in only $20 \%$ of the MMP-9-/-/MMP- $12^{-/-}$animals, compared with a combined prevalence of $94 \%$ in wildtype background controls ( $P<0.05, \chi^{2}$ analysis). In contrast, aneurysm development was unaffected in mice with isolated deficiency of MMP-12, which exhibited a mean increase in aortic diameter of $134 \%( \pm 7 \%)$ and a $100 \%$ incidence of AAAs. The expected molecular alterations in these gene-targeted mice were confirmed by both substrate zymography and RT-PCR/Southern blot analysis (Figure 4, b and c), with no evidence for a "compensatory" increase in one elastolytic enzyme in the absence of the other.

Light microscopy revealed that the reduction in aneurysmal dilatation in MMP-9-deficient mice was associated with preservation of the medial elastic lamellae (Figure 5). Thus, at 14 days after perfusion the elastic lamellae were well preserved in MMP-9-/- and MMP-9-/-/MMP-12-/- mice, whereas MMP-12/- mice exhibited destruction of medial elastic fibers equivalent to that observed in wild-type animals. There was also no detectable suppression of the elastase-induced inflammatory response in any of the genetically altered mice; in particular, MMP-9-/- animals continued to display aortic wall infiltration by mononuclear phagocytes and neutrophils. Similar morphologic features were observed in wild-type mice treated with doxycycline, a finding consistent with pharmacologic inhibition of metalloproteinases within the aortic wall.
Effects of bone marrow transplantation on the aneurysmresistant phenotype. To determine if the aneurysm-resistant phenotype observed in MMP-9-/- mice is attributable to the absence of MMP-9 in mononuclear phagocytes or neutrophils, elastase perfusion was performed in animals that had been subjected to lethal irradiation and syngeneic bone marrow transplantation (Table 2). Compared with $129 / \mathrm{SvEv}$ controls reconstituted with wild-type bone marrow, there was a significant reduction in aortic dilatation in wild-type mice reconstituted with bone marrow from MMP-9-/donors. In contrast, MMP-9-/- animals reconstituted with wild-type bone marrow had a significantly greater extent of aortic dilatation than controls reconstituted from MMP-9-/- donors. These results provide evidence
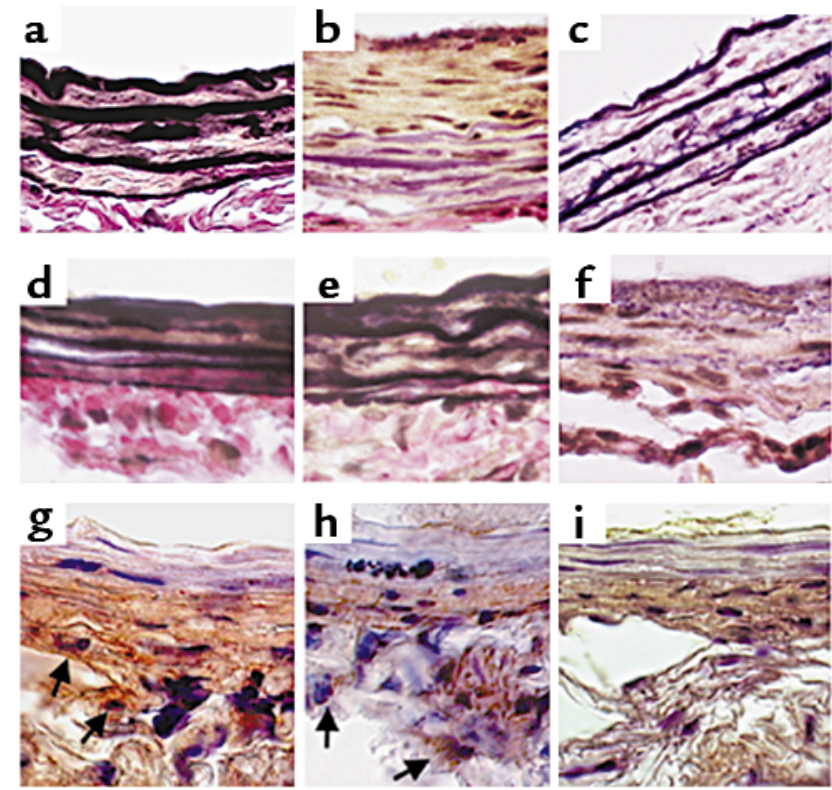

\section{Figure 5}

Histologic changes during the suppression of elastase-induced aneurysmal dilatation. (a-h) Sections stained with WVG for elastin. Normal preperfusion aorta (a), 14 days postperfusion wild-type mice with aneurysmal dilatation (b), 14 days postperfusion wild-type mice treated with doxycycline (c), 14 days postperfusion MMP-9-/- mice (d), 14 days postperfusion MMP-9-/-/MMP-12-/- mice (e), 14 days postperfusion MMP-12-/- mice (f). (g-i) Fourteen days postperfusion MMP-9-/- mice, with immunoperoxidase staining for macrophages $(\mathbf{g})$, neutrophils $(\mathbf{h})$, and mouse $\operatorname{lgG}$ (negative control) (i). $\times 100$. 
that the aneurysm-resistant phenotype in MMP-9-/mice is due to a lack of expression of MMP-9 in infiltrating inflammatory cells rather than developmental abnormalities in aortic wall structure or the absence of MMP-9 expression by resident aortic wall cell types.

\section{Discussion}

Aneurysmal degeneration involves destructive remodeling of aortic wall connective tissues associated with chronic mononuclear inflammation and excessive local production of matrix-degrading proteinases. Although pancreatic elastase is not known or suspected to play a role in human AAAs, elastase-induced animal models have been valuable for examining biological mechanisms involved in aneurysm disease. In the studies reported here, we show that transient perfusion of the mouse aorta with a low concentration of elastase does not cause immediate aneurysmal dilatation, but initiates a pathologic response that consistently results in the delayed development of AAAs. Because late aneurysmal dilatation was not observed after perfusion with heat-inactivated elastase, aneurysm development is initially dependent on elastase activity and the subsequent inflammatory response, rather than simply the mechanical effects of intraluminal perfusion or an immunologic reaction to foreign (porcine) protein. In addition to submicroscopic injury to elastic fibers, one of the consequences of transient elastase perfusion may be to generate proteolytic fragments derived from elastin, laminin, and other matrix proteins. In high local concentrations, these biologically active peptides may promote leukocyte chemotaxis and increased MMP expression $(23,24)$. The response of the elastaseinjured mouse aorta therefore parallels that which occurs in the rat, recapitulating many of the tissueremodeling events thought to be important in the pathophysiology of human AAAs.

Based on evidence that tetracycline derivatives suppress MMP activity by both direct and indirect mechanisms (25), we used doxycycline to examine if elevated MMP production might be functionally significant in the development of elastase-induced AAAs. Similar to our previous studies in rats using either doxycycline, nonantibiotic chemically modified tetracyclines or hydroxamate-based MMP inhibitors $(16,17,19)$, treatment with doxycycline attenuated elastase-induced aneurysmal degeneration in the mouse by approximately $50 \%$. This was associated with preservation of the elastic lamellae despite the presence of infiltrating inflammatory cells, suggesting that doxycycline exerts its effects by inhibiting proteinases responsible for connective tissue destruction. Even though it remains unclear if direct pharmacologic inhibition or a reduction in gene expression is most important in the MMPinhibiting effects of tetracyclines in vivo, we found recently that doxycycline can suppress aortic wall expression of MMP-9 up to fivefold in patients undergoing elective AAA repair (26).

The most significant aspect of this study was the application of the elastase-induced murine model to gene-targeted animals, allowing us to investigate the relative contributions of MMP-9 and MMP-12, two elastolytic MMPs previously implicated in human AAAs. Although we found that aortic wall expression of both metalloproteinases is upregulated in wild-type mice within 7 to 14 days of elastase perfusion, animals deficient in MMP-9 (either in isolation or in combination with MMP-12) exhibited a marked reduction in aortic dilatation. The suppression of aneurysms in MMP-9-deficient mice was associated with morphologic preservation of the elastic lamellae despite the presence of infiltrating mononuclear phagocytes and neutrophils, suggesting a direct role for MMP-9-mediated proteolysis in AAAs. These observations also demonstrate for the first time that a single metalloproteinase, MMP-9, is required for the development of elastase-induced AAAs.

Even though it can be inferred from this study that MMP-9 participates in aneurysm development by virtue of its elastolytic activity (27), MMP-9 may fulfill only one of several critical steps in accelerated elastic fiber degradation. The possibility that other gene products may also be required is supported by earlier studies showing that urokinase-type plasminogen activator (u-PA) and MMPs are both important for aneurysm formation in ApoE-deficient mice (28), that overexpression of plasminogen activator inhibitor-1 can suppress aneurysm formation and rupture in rat xenografts (29), and that several other metalloproteinases are overexpressed in human AAAs (30). Thus, extracellular activation of proMMP-9 may depend on additional enzymes, such as MMP-3 (stromelysin-1), u-PA/plasmin, or neutrophil elastase (31-33), and other proteinases may augment MMP-9-mediated degradation of elastic fibers (e.g., MMP-2, MMP-12, neutrophil elastase, or elastolytic cathepsins) $(7,34)$. Finally, it is also important to consider how genetic deficiency in MMP-9 might have altered the function of tissue inhibitors of metalloproteinases (TIMPs) during the process of elastase-induced aneurysmal degeneration. MMP-9 is secreted from most cell types in a complex with TIMP-1 (35), and whereas it is reasonable to expect that TIMP-1 is also expressed within the elastaseinjured aorta, it is not yet known how MMP-9 deficiency influences TIMP-1 expression, secretion, or its biological availability to interact with other MMPs.

We found that isolated genetic deficiency of MMP-12 does not have a significant influence on elastic fiber degradation or aneurysm development, indicating that MMP-12 is not essential for elastase-induced AAAs and that its expression cannot "compensate" for the absence of MMP-9. This was surprising given that MMP-12 appears to play a critical role in macrophagemediated elastic fiber degradation in vitro (21) and in experimental pulmonary emphysema (22), but emphasizes that individual metalloenzymes may have unique roles in tissue-specific inflammatory responses (36). 
Because our studies did reveal a slight enhancement of the aneurysm-resistant phenotype in double-deficient mice compared with those with MMP-9 deficiency alone, it appears that MMP-12 might still be acting to assist elastic fiber degradation in this model. Further studies will be needed to examine this possibility.

Our immunohistochemical studies revealed that inflammatory cells, particularly mononuclear phagocytes, are the dominant source of MMP-9 during elastaseinduced aneurysmal degeneration. Studies employing in situ hybridization have also shown that "aneurysm-infiltrating macrophages" are the principal source of MMP-9 in human AAAs (8). Although neutrophils can store and release MMP-9 from specific granules upon stimulation, PMNs do not actively synthesize MMP-9 mRNA, and this enzyme is not necessary for PMN emigration into tissue $(36,37)$. The presence of neutrophils within the elastaseinjured aortic wall therefore suggests that they may be one of the initial sources of MMP-9 in our model, but that other cell types are likely responsible for sustained MMP-9 expression. Whereas vascular smooth muscle cells and adventitial fibroblasts produce only MMP-2 under normal conditions, both of these cell types can also express MMP-9 in pathologic states or upon activation by proinflammatory cytokines. Our studies confirm that MMP-9 is not expressed to a significant degree in the normal mouse aorta, but that it increases early after elastase perfusion and even more so between 7 and 14 days. This coincides with leukocyte infiltration of the elastaseinjured aorta, supporting the view that mononuclear phagocytes are responsible for the increased production of MMP-9 at later intervals. More convincing evidence that inflammatory cells are responsible for MMP-9 expression arises from our studies in mice subjected to irradiation and bone marrow transplantation, which demonstrated that the aneurysm-resistant phenotype in MMP-9-/- mice can be effectively "rescued" by reconstitution with wild-type bone marrow. Because the aneurysm-resistant phenotype can also be transferred to wild-type mice through MMP-9-/- hematogenous cells, the amount of MMP-9 that might be expressed by resident aortic wall cells appears to be insufficient to sustain aneurysmal degeneration.

Population-based screening studies reveal that up to 9 percent of individuals over the age of 65 harbor an unsuspected AAA (38). Although the vast majority of these lesions are small and asymptomatic, their natural history is characterized by progressive growth and eventual rupture. Recognition that different MMPs participate in the pathobiology of AAAs has fostered the notion that these enzymes might be useful therapeutic targets in this disease, providing a novel approach to reduce the degradation of fibrillar proteins responsible for maintaining aortic wall tensile strength and potentially suppressing the rate of aneurysm expansion (39). The present study provides new experimental evidence in support of this concept and suggests that strategies targeting MMP-9 might be a particularly fruitful avenue for further investigation.

\section{Acknowledgments}

Supported by National Heart, Lung, and Blood Institute grants HL-29594 (S.D. Shapiro), HL-47328 (R.M. Senior), and HL-56701 (R.W. Thompson); the Parker B. Francis Fellowship Program (J.M. Shipley); the Alan A. and Edith L. Wolff Charitable Trust (R.M. Senior); and the Wylie Scholar Award from the Pacific Vascular Research Foundation, San Francisco, California (R.W. Thompson). We thank William C. Parks (Washington University, St. Louis, Missouri, USA) for valuable discussions.

1. Cole, C.W., et al. 1994. Proceedings of the workshop on the control of abdominal aortic aneurysm. Chronic Dis. Can. 15(Suppl.):S1-S64.

2. Thompson, R.W. 1996. Basic science of abdominal aortic aneurysms: emerging therapeutic strategies for an unresolved clinical problem. Curr. Opin. Cardiol. 11:504-518.

3. Dobrin, P.B., and Mrkvicka, R. 1994. Failure of elastin or collagen as possible critical connective tissue alterations underlying aneurysmal dilatation. Cardiovasc. Surg. 2:484-488.

4. Shah, P.K. 1997. Inflammation, metalloproteinases, and increased proteolysis: an emerging pathophysiological paradigm in aortic aneurysm. Circulation. 96:2115-2117.

5. Parks, W.C., Pierce, R.A., Lee, K.A., and Mecham, R.P. 1993. Elastin. Advances in Molecular Cell Biology. 6:133-182.

6. Freestone, T., et al. 1995. Inflammation and matrix metalloproteinases in the enlarging abdominal aortic aneurysm. Arterioscler. Thromb. Vasc. Biol. 15:1145-1151.

7. Davis, V., et al. 1998. Matrix metalloproteinase-2 production and its binding to the matrix are increased in abdominal aortic aneurysms. Arterioscler. Thromb. Vasc. Biol. 18:1625-1633.

8. Thompson, R.W., et al. 1995. Production and localization of 92-kilodalton gelatinase in abdominal aortic aneurysms: an elastolytic metalloproteinase expressed by aneurysm-infiltrating macrophages. J. Clin. Invest. 96:318-326.

9. Curci, J.A., Liao, S., Huffman, M.D., Shapiro, S.D., and Thompson, R.W. 1998. Expression and localization of macrophage elastase (matrix metalloproteinase-12) in abdominal aortic aneurysms. J. Clin. Invest. 102:1900-1910

10. McMillan, W.D., et al. 1997. Size matters: the relationship between MMP-9 expression and aortic diameter. Circulation. 96:2228-2232.

11. McMillan, W.D., and Pearce, W.H. 1999. Increased plasma levels of metalloproteinase- 9 are associated with abdominal aortic aneurysms. J. Vasc. Surg. 29:122-127.

12. Dobrin, P.B. 1999. Animal models of aneurysms. Ann. Vasc. Surg. 13:641-648.

13. Anidjar, S., et al. 1990. Elastase induced experimental aneurysms in rats. Circulation. 82:973-981.

14. Anidjar, S., Dobrin, P.B., Eichorst, M., Graham, G.P., and Chejfec, G. 1992. Correlation of inflammatory infiltrate with the enlargement of experimental aortic aneurysm. J. Vasc. Surg. 16:139-147.

15. Halpern, V.J., et al. 1994. The elastase infusion model of experimental aortic aneurysms: synchrony of induction of endogenous proteinases with matrix destruction and inflammatory cell response. J. Vasc. Surg. 20:51-60.

16. Petrinec, D., et al. 1996. Doxycycline inhibition of aneurysmal degeneration in an elastase-induced rat model of abdominal aortic aneurysm: preservation of aortic elastin associated with suppressed production of $92 \mathrm{kD}$ gelatinase. J. Vasc. Surg. 23:336-346.

17. Curci, J.A., Petrinec, D., Liao, S.X., Golub, L.M., and Thompson, R.W. 1998. Pharmacologic suppression of experimental abdominal aortic aneurysms: a comparison of doxycycline and four chemically modified tetracyclines. J. Vasc. Surg. 28:1082-1093.

18. Bigatel, D.A., et al. 1999. The matrix metalloproteinase inhibitor BB-94 limits expansion of experimental abdominal aortic aneurysms. J. Vasc. Surg. 29:130-138.

19. Moore, G., et al. 1999. Suppression of experimental abdominal aortic aneurysms by systemic treatment with a hydroxamate-based matrix metalloproteinase inhibitor (RS 132908). J. Vasc. Surg. 29:522-532.

20. Vu, T.H., et al. 1998. MMP-9/gelatinase B is a key regulator of growth plate angiogenesis and apoptosis of hypertrophic chondrocytes. Cell. 93:411-422.

21. Shipley, J.M., Wesselschmidt, R.L., Kobayashi, D.K., Ley, T.J., and Shapiro, S.D. 1996. Metalloelastase is required for macrophage-mediated proteolysis and matrix invasion in mice. Proc. Natl. Acad. Sci. USA. 93:3942-3946.

22. Hautamaki, R.D., Kobayashi, D.K., Senior, R.M., and Shapiro, S.D. 1997. Requirement for macrophage elastase for cigarette smoke-induced emphysema in mice. Science. 277:2002-2004. 
23. Senior, R.M., Griffin, G.L., and Mecham, R.P. 1980. Chemotactic activity of elastin-derived peptides. J. Clin. Invest. 66:859-862.

24. Corcoran, M.L., Kibbey, M.C., Kleinman, H.K., and Wahl, L.M. 1995. Laminin SIKVAV peptide induction of monocyte/macrophage prostaglandin E2 and matrix metalloproteinases. J. Biol. Chem. 270:10365-10368.

25. Golub, L.M., et al. 1998. Tetracyclines inhibit connective tissue breakdown by multiple non-antimicrobial mechanisms. Adv. Dent. Res. 12:12-26.

26. Curci, J.A., et al. 2000. Preoperative treatment with doxycycline reduces aortic wall expression and activation of matrix metalloproteinases in patients with abdominal aortic aneurysms. J. Vasc. Surg. 31:325-342.

27. Senior, R.M., et al. 1991. Human 92- and 72-kilodalton type IV collagenases are elastases. J. Biol. Chem. 266:7870-7875

28. Carmeliet, P., et al. 1997. Urokinase-generated plasmin activates matrix metalloproteinases during aneurysm formation. Nat. Genet. 17:439-444

29. Allaire, E., et al. 1998. Prevention of aneurysm development and rupture by local overexpression of plasminogen activator inhibitor-1. Circulation. 98:249-255.

30. Thompson, R.W., and Parks, W.C. 1996. Role of matrix metalloproteinases in abdominal aortic aneurysms. Ann. NY Acad. Sci. 800:157-174.

31. Shapiro, S.D., et al. 1995. Activation of the 92-kDa gelatinase by stromelysin and 4-aminophenylmercuric acetate. Differential processing and stabilization of the carboxyl-terminal domain by tissue inhibitor of metalloproteinases (TIMP). J. Biol. Chem. 270:6351-6356.

32. Mazzieri, R., et al. 1997. Control of type IV collagenase activity by com- ponents of the urokinase-plasmin system: a regulatory mechanism with cell-bound reactants. EMBOJ. 16:2319-2332.

33. Ferry, G., et al. 1997. Activation of MMP-9 by neutrophil elastase in an in vivo model of acute lung injury. FEBS Lett. 402:111-115.

34. Sukhova, G.K., Shi, G.P., Simon, D.I., Chapman, H.A., and Libby, P. 1998. Expression of the elastolytic cathepsins $\mathrm{S}$ and $\mathrm{K}$ in human atheroma and regulation of their production in smooth muscle cells. J. Clin. Invest. 102:576-583.

35. Goldberg, G.I., Strongin, A., Collier, I.E., Genrich, L.T., and Marmer, B.L. 1992. Interaction of $92-\mathrm{kDa}$ type IV collagenase with the tissue inhibitor of metalloproteinases prevents dimerization, complex formation with interstitial collagenase, and activation of the proenzyme with stromelysin. J. Biol. Chem. 267:4583-4591.

36. Betsuyaku, T., Shipley, J.M., Liu, Z., and Senior, R.M. 1999. Neutrophil emigration in the lungs, peritoneum, and skin does not require gelatinase B. Am. J. Respir. Cell Mol. Biol. 20:1303-1309.

37. Liu, Z., et al. 1998. Gelatinase B-deficient mice are resistant to experimental bullous pemphigoid. J. Exp. Med. 188:475-482.

38. Alcorn, H.G., Wolfson, S.K., Jr., Sutton-Tyrrell, K., Kuller, L.H., and O'Leary, D. 1996. Risk factors for abdominal aortic aneurysms in older adults enrolled in The Cardiovascular Health Study. Arterioscler. Thromb. Vasc. Biol. 16:963-970.

39. Thompson, R.W., and Baxter, B.T. 1999. MMP inhibition in abdominal aortic aneurysms: rationale for a prospective randomized clinical trial. Ann. NY Acad. Sci. 878:159-178. 